\title{
COMPUTATIONAL MAGNETOHYDRODYNAMIC MODEL OF AN ADVANCED FUSION FUEL GAS DYNAMIC MIRROR PROPULSION SYSTEM
}

\author{
Chad J. Ohlandt*, Dept. of Aerospace Engineering \\ Terry Kammash ${ }^{\dagger}$, Dept. of Nuclear Engineering \\ Kenneth G. Powell ${ }^{\ddagger}$, Dept. of Aerospace Engineering \\ University of Michigan, Ann Arbor, MI 48109
}

\begin{abstract}
$\underline{\text { Abstract }}$
In support of the development of gas dynamic mirror (GDM)[1] machines as space propulsion systems for interplanetary flight, a computational modeling tool is being developed to corroborate analytical models and to guide future experimental work. In this paper the structure of an engineering and computational design study of a gas dynamic mirror (GDM) fusion propulsion system powered by the advanced fusion fuel, $p-{ }^{11} B$, is presented. The conclusion is that $Q>1$, homogeneous plasma GDMs with a direct mission trajectory are impractical with $p-{ }^{11} B$ due to bremsstrahlung radiation losses. This reinforces the need for a 3-D complex geometry ideal magnetohydrodynamic modeling tool[2] based on finite volume methods and adaptive Cartesian grids with a new implicit algorithm to deal with the extreme magnetoacoustic wave speeds generated by the magnetic fields of the GDM machine.
\end{abstract}

\section{Introduction}

Traditional fusion research efforts have discarded magnetic mirrors as a viable option due to the plasma losses from the open configuration. Fortunately, the primary design criteria of a plasma propulsion system is significant plasma "loss" which results in thrust. Taking advantage of this, Kammash et al. designed a gas dynamic mirror fusion propulsion system. $[1,3,4]$ While potentially feasible, the resulting GDM configurations had masses of 400-1000 metric tons (1-2.5 International Space Station Alphas for comparison) and are unlikely to be launched into space in the near future. Up to $75 \%$ of the GDM mass budget is devoted to thermal converters and radiators to eliminate waste heat primarily from neutrons produced by the fusion of the deuterium-tritium $(D-T)$ fuel. A reduction in neutron production would significantly decrease the GDM mass requirements. Additionally, high energy neutrons require additionally shielding to protect system components. Finally, tritium is a radioactive fuel which creates safety concerns during launch into orbit or possible re-entry into the Earth's atmosphere. This work focuses on two possibilities to address these issues: advanced fusion fuels and/or assisted reactor systems $(Q<1)$.

Again, traditional fusion research has studied advanced fusion fuels such as $D-{ }^{3} \mathrm{He},{ }^{3} \mathrm{He}-{ }^{3} \mathrm{He}$, and $p-{ }^{11} B$ which all generate lower levels of neutron production or none at all. They have been generally been regarded as unsuitable for low $\beta$ reactors such as the popular tokamak configuration and uneconomical for power production due to radiation power losses and limited Q-values. $[5,6,7,8,9]$ However, these fuels could be ideal for a high- $\beta$ GDM propulsion system. While $\mathrm{Q}$ only needs to exceed breakeven and radiation can be used for thrust enhancement[3], the aneutronic nature of the fuels could reduce the weight of a GDM propulsion system. Such a revolutionary system that is not limited by the traditional assumptions of the fusion research establishment would open the solar system to exploration and development.

Using advanced fusion fuels with no or minimal neutron production would reduce the waste heat and therefore the mass of the thermal converters and radiators. Table 1 lists various fusion fuels and their corresponding parameters. The aneutronic ${ }^{3} \mathrm{He}-{ }^{3} \mathrm{He}$ and $p-{ }^{11} B$ reactions avoid neutron energy loss, but

\footnotetext{
${ }^{*}$ Graduate Student, Member AIAA

†Professor, Associate Fellow AIAA

$\ddagger$ Professor, Member AIAA

Copyright (C)2002 by Chad Ohlandt. Published by the American Institute of Aeronautics and Astronautics, Inc., with permission.
} 
require much larger ignition temperatures. The compromise reaction, $\mathrm{D}-{ }^{3} \mathrm{He}$, has been studied and offers lower relative neutron power levels, but the resulting configuration is significantly more massive the the original $D-T$ concept. As such, $p-{ }^{11} B$ is the most promising for further study.

\section{Proton-Boron 11 GDMs}

The $p-{ }^{11} B$ appears very attractive given its largely aneutronic nature, availability, and reasonable ignition temperature. However, the primary challenge of advanced fusion fuels are the higher plasma temperatures. At these temperatures, bremsstrahlung and synchrotron radiation losses become so significant as to limit the $\mathrm{Q}$ factor or even the ability to reach ignition. Previously developed parametric models $[1,3]$ with $p-{ }^{11} B$ parameters were modified to take into account that $T_{e}$ is not equal to $T_{i}$, using Dawson[10] to find it, and to calculate bremsstrahlung power as does Nevins[11]. The important characteristics of this model include a high density (Maxwellian plasma) in a large aspect ratio GDM with homogeneous properties throughout, a Q of slightly greater than 1 to account for efficiency losses, and a mission trajectory which assumes a direct line from origin to destination with constant acceleration or deceleration during transit.

The charts in Figure 1 indicate radiation powers as multiples of the fusion power generated. With a reflectivity of .9 , the synchrotron power is too much. Increasing the reflectivity to .99 , a potential $p-{ }^{11} B$ system would work around $160 \mathrm{keV}$ giving an optimal balance between bremsstrahlung and synchrotron losses. However, a $Q>1$ is only possible when $P_{\text {rad }} / P_{f}$ ratio is below 2 . In the case of .9999 reflectivity, synchrotron radiation becomes unimportant. It should be noted that bremsstrahlung radiation is heavily concentrated in the x-ray band and cannot be easily reflected. In the remaining cases, $\mathrm{T}=300 \mathrm{keV}$ and $\mathrm{R}=.9999$ were assumed. Even in this best case scenario, radiation losses are a multiple of of the fusion power (roughly 1.8) indicating the ignition is not feasible the GDM will function only in a driven mode. Fortunately, high $Q$ is not necessary for GDM operation, only slightly greater than 1 is needed.

Figure 2 explores the affects of plasma density on our GDM propulsion system. Greater density increases total fusion power and thrust, but it also increases the radiation load with corresponding thermal converters and radiators. The first chart indicates on optimum density of $2.0 * 10^{16}$ particles per cubic centimeter the leads to the minimum dry mass of the system as well as approaching the best trip time. The second chart explains this by showing how the thermal radiator mass dominates the system above this density.

The fuel ratio, $\frac{n_{B}}{n_{p}}$, also has a significant effect on the GDM system. The primary driver of this is the much greater charge of boron atoms $(\mathrm{Z}=5)$. ${ }^{11} B$ contributes, on a per atom basis, much more to bremsstrahlung radiation than protons. Additionally, it also adds to the electron density increasing the synchrotron radiation. Of course, too little ${ }^{11} B$ reduces the fusion power. Figure 3 clearly indicates that a fuel ratio of .15 produces the optimal dry mass and trip time.

With the various system parameters optimized, the actual size of the system is directly a function of its radius which is specified through the mirror radius. Dry mass and trip time are plotted against the mirror radius in Figure 3. While the smaller dimension continues to reduce the total mass, the diminishing thrust increases travel time. Choosing a mirror radius of .005 meters give close to optimal trip time with minimum mass.

As such, the best system possible requiring $Q>1$ is listed in Table 2. Unfortunately, such a system is 24 kilometers long, weighing over a million metric tons, and takes a year and half to reach Mars. Fundamentally, the large bremsstrahlung losses increase system size beyond a practical limit to achieve a $Q>1$ design.

\section{Nuclear Electric Assisted}

If the $Q>1$ requirement is relaxed and supplemental power is generated with a nuclear electric fission reactor, the size of the system can be reduced. By introducing supplemental power as a fraction of the fusion power and adding the mass of an advanced nuclear electric space power system based on Smith[12], the reduced system parameters can be found in Figure 4. For a $\mathrm{T}=300 \mathrm{keV}$ system, the effects are moderate overall. However, with $Q<1$ being acceptable, the temperature can be reduced to an optimal $160 \mathrm{keV}$. Also shown in Figure 4, the impact form nuclear electric assist are significant on the system size and mass. Nonetheless, the system remains unsuitably large.

\section{Conclusions}

The radiative losses from bremsstrahlung radiation in $p-{ }^{11} B$ GDM system are too great to develop a practical system with a homogeneous plasma and 
a direct mission trajectory. Even if you relax the expectation of $Q>1$ and assist with nuclear electric power, the system size and mass remain too large. Nonetheless, the aneutronic nature of advanced fuels, availability, and non-radioactive nature of advanced fusion fuels remains attractive. If such a system is to be developed, it will require a non-uniform plasma at lower temperatures and a computational MHD model to evaluate it. Further issues worth exploring are thrust enhancement and alternate mission trajectories.

\section{Future Work}

The authors previously have developed a MHD model for a GDM system[2]. The code has three major components, the geometry constructor, a grid generator, and the flow solver. The geometry constructor uses various simple shapes that can be rotated and extruded to produce complex 3-D geometries. The geometries consist of polygon mesh surfaces which are combined to create three dimensional objects. Initial grids are generated in a matter of hours using automated, Cartesian methods, and solution-based adaption allows the code to increase resolution around flow regions of interest during the flow solution. A finite volume conservation formulation is the basis of the various MHD solvers implemented.[13]

However, the explicit nature of the algorithm was not effective at dealing with Aflven wave speeds that approach a few percent the speed of light. As such, an improved version of the code using a fully implicit solver is being developed. This will allow the concept of non-uniform plasma GDM systems to be explored.

\section{References}

[1] Kammash, T. and Lee, M.-J., "Gasdynamic Fusion Propulsion System for Space Exploration", Journal of Propulsion and Power 11, 3, pp. 544$53,1995$.

[2] Ohlandt, C. J., Kammash, T., and Powell, K. G., "Computational magnetohydrodynamic model of a gasdynamic mirror propulsion system," AIAA Paper 99-2702, AIAA/ASME/SAE/ASEE Joint Propulsion Conference and Exhibit, 35th, Los Angeles, CA, June 20-24, 1999.

[3] Kammash, T., Lee, M.-J., and Poston, D.I., "High-Thrust-High-Specific Impulse Gasdynamic
Fusion Propulsion System", Journal of Propulsion and Power 13, 3, pp. 421-7, 1997.

[4] Kammash, T. and Galbraith, D.L., "Improved Physics Model for the Gasdynamic Fusion Propulsion System", Journal of Propulsion and Power 14, 1, pp. 24-8, 1998.

[5] McNally, J.R., "Physics of Fusion Fuel Cycles", Nuclear Technology/Fusion, 2, pp. 9-28, 1982.

[6] Kernbichler, W., Feldbacher, R., and Heindler, M., "Parametric Analysis of p-B11 as Advanced Reactor Fuel", Proc. 10th Int. Conf. Plasma Physics and Controlled Nuclear Fusion Research (1984), Vol. 3, pp. 429-40, 1987.

[7] Heindler, M., and Kernbichler, W., "Advance Fuel Fusion", Proceedings of the Fifth International Conference on Emerging Nuclear Energy Systems, pp. 177-82, 1989.

[8] Best, R.W., "Advanced Fusion Fuel Cycles", Fusion Technology, 17, 1990.

[9] Perkins, L.J., Hammer, J.H., and Drake, R.P., "Fusion, the Competition, and the Prospects for Alternative Fusion Concepts", Current Trends in International Fusion Research, ed. by Panarella, Chap. 23, 1997.

[10] Dawson, J.M., "Advanced Fusion Reactors", $F u$ sion (Vol 1. Part B), ed. by Teller, Academic Press, New York, pp. 453-501, 1981.

[11] Nevins, W.M., "A Review of Confinement Requirements for Advanced Fuels", Journal of Fusion Energy, Vol. 17, No. 1, pp. 25-32, 1998

[12] Smith, B.M., Knight, T.W., Anghaie, S., "Overview of Nuclear MHD Power Conversion For Multi-Megawatt Electric Propulsion", Space Technology and Applications International Forum-2001, ed. by El-Genk, American Institute of Physics, pp. 916-922, 2001.

[13] Powell, K.G., Roe, P. L., Linde, T. J., Gombosi, T. I., and De Zeeuw, D. L., "A Solution-Adaptive Upwind Scheme for Ideal Magnetohydrodynamics", Journal of Computational Physics, 153, pp. 284-309, 1999. 


\begin{tabular}{|c|c|c|c|c|}
\hline Fuel & Products & $\begin{array}{c}\text { Total } \\
\text { Energy [MeV] }\end{array}$ & $\begin{array}{c}\text { Charged Particle } \\
\text { Energy [MeV] }\end{array}$ & $\begin{array}{c}\text { Optimal Ignition } \\
\text { Temperature [keV] }\end{array}$ \\
\hline$D-T$ & $n+{ }^{4} \mathrm{He}$ & 17.6 & 3.5 & 10.5 \\
$D-D$ & $p+T$ & 4.0 & 4.0 & 15 \\
& $n+{ }^{3} \mathrm{He}$ & 3.3 & .8 & 15 \\
$D-{ }^{3} \mathrm{He}$ & $p+{ }^{4} \mathrm{He}$ & 18.3 & 18.3 & 60 \\
${ }^{3} \mathrm{He}-{ }^{3} \mathrm{He} e$ & $2 p+{ }^{4} \mathrm{He}$ & 12.9 & 12.9 & 1000 \\
$p-{ }^{11} \mathrm{~B}$ & $3{ }^{4} \mathrm{He}$ & 8.7 & 8.7 & 150 \\
\hline
\end{tabular}

Table 1: Table of fusion fuels with relevant parameters.
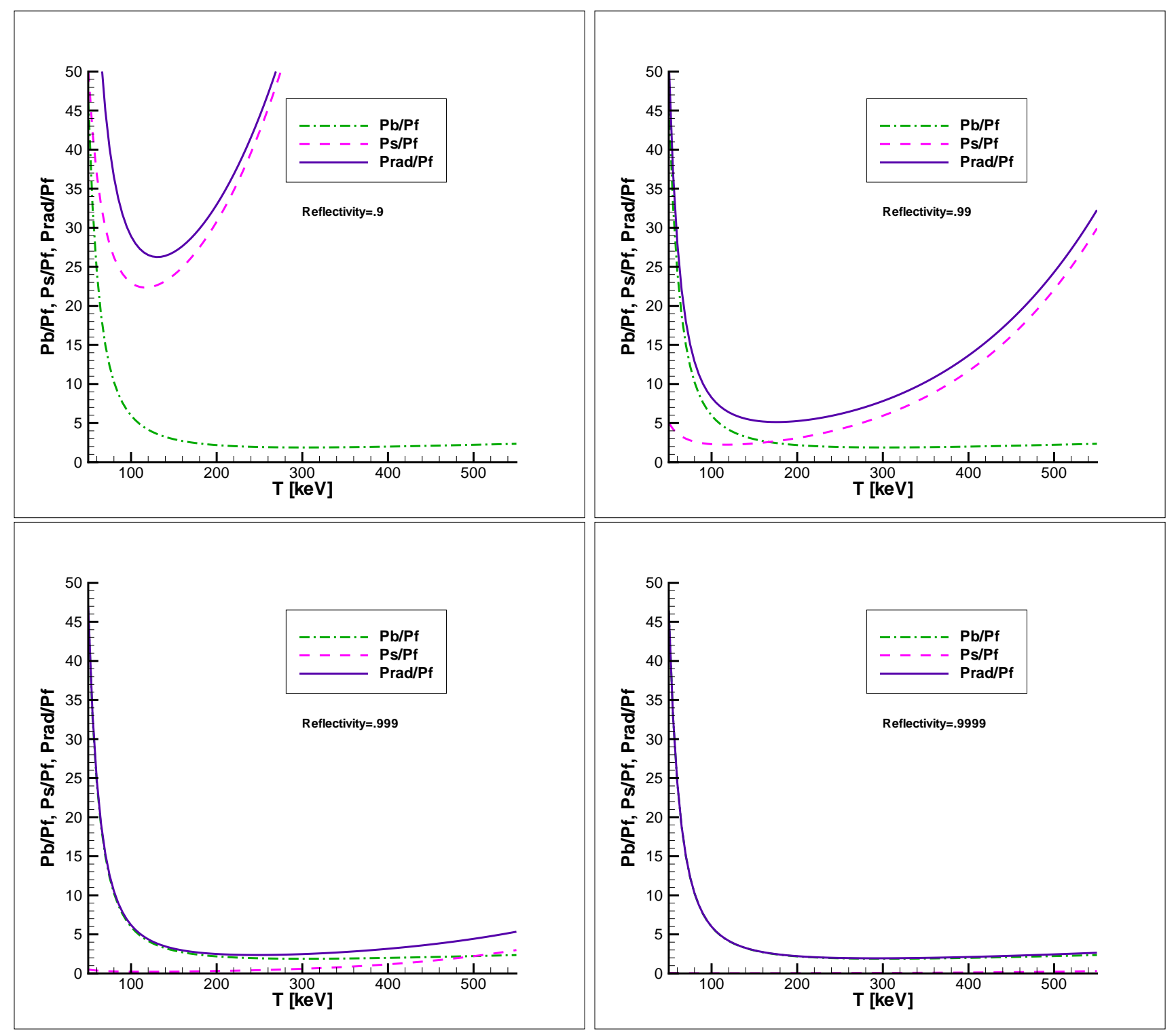

Figure 1: Power ratios as a function of temperature for different reflectivities $(.9, .99, .999, .9999) . P_{b}$ is bremsstrahlung radiation power, $P_{s}$ is synchrotron radiation power, and $P_{r a d}$ is $P_{b}+P_{s}$. 


\begin{tabular}{|c|c|}
\hline Reaction Type & $p-{ }^{11} \mathrm{~B}$ \\
Plasma Density & $2 \mathrm{e}+16 \mathrm{\#} / \mathrm{cm}^{3}$ \\
Hydrogen Density & $1.74 \mathrm{e}+16 \mathrm{\#} / \mathrm{cm}^{3}$ \\
Boron-11 Density & $2.61 \mathrm{e}+15 \mathrm{\#} / \mathrm{cm}^{3}$ \\
Electron Density & $3.04 \mathrm{e}+16 \mathrm{\#} / \mathrm{cm}^{3}$ \\
Plasma Temperature & $300 \mathrm{keV}$ \\
Beta (vacuum) & 0.95 \\
Plasma Mirror Ratio & 100 \\
Plasma Mirror Radius & $0.005 \mathrm{~m}$ \\
Halo Thickness & $0.1 \mathrm{~m}$ \\
Shield Magnet Gap & $0.1 \mathrm{~m}$ \\
Shield Thickness & $0.19 \mathrm{~m}$ \\
Injector Eff. & 1 \\
Thermal Conv. Eff. & 0.45 \\
Direct Conv. Eff. & 0.9 \\
Magnet Current Den. & $2.5 \mathrm{e}+08 \mathrm{MA} / \mathrm{m}^{2}$ \\
Destination Mars & $7.8 \mathrm{e}+10 \mathrm{~m}$ \\
Calculated Parameters- & \\
Vacuum magnetic field, Bp0 & $80.07 \mathrm{Tesla}$ \\
Gain Factor, Q & 1.22222 \\
Plasma Length, L & $23635 \mathrm{~m}$ \\
Injection Energy, Ein & $1464.34 \mathrm{keV}$ \\
Loss Energy, EL & $600 \mathrm{keV}$ \\
Thrust & $150985 \mathrm{~N}$ \\
Thrust Power & $4.44 \mathrm{e}+05 \mathrm{MW}$ \\
Injection Power & $2.17 \mathrm{e}+06 \mathrm{MW}$ \\
Fusion Power & $2.65 \mathrm{e}+06 \mathrm{MW}$ \\
Bremsstrahlung Power & $4.67 \mathrm{e}+06 \mathrm{MW}$ \\
Synchrotron Power & $1.67 \mathrm{e}+05 \mathrm{MW}$ \\
Total Dry Mass & $1.28+06 \mathrm{mT}$ \\
Engine Mass Fraction & 0.29 \\
Converters Mass Fraction & 0.30 \\
Radiator Mass Fraction & 0.41 \\
Isp & $693791 \mathrm{~s}$ \\
Round Trip Time & $3.27 \mathrm{years}$ \\
Trip Time AB & $1.63 \mathrm{years}$ \\
Fusion Power & $1.43 \mathrm{e}+10 \mathrm{watts} / \mathrm{m}^{3}$ \\
Bremsstrahlung Power & $2.51 \mathrm{e}+10 \mathrm{watts} / \mathrm{m}^{3}$ \\
Pb/Pf & 1.76 \\
Synchrotron Power & $8.97 \mathrm{e}+08 \mathrm{watts} / \mathrm{m}^{3}$ \\
Ps/Pf & 0.06 \\
\hline
\end{tabular}

Table 2: Optimized $Q>1 p-{ }^{11} B$ GDM. 

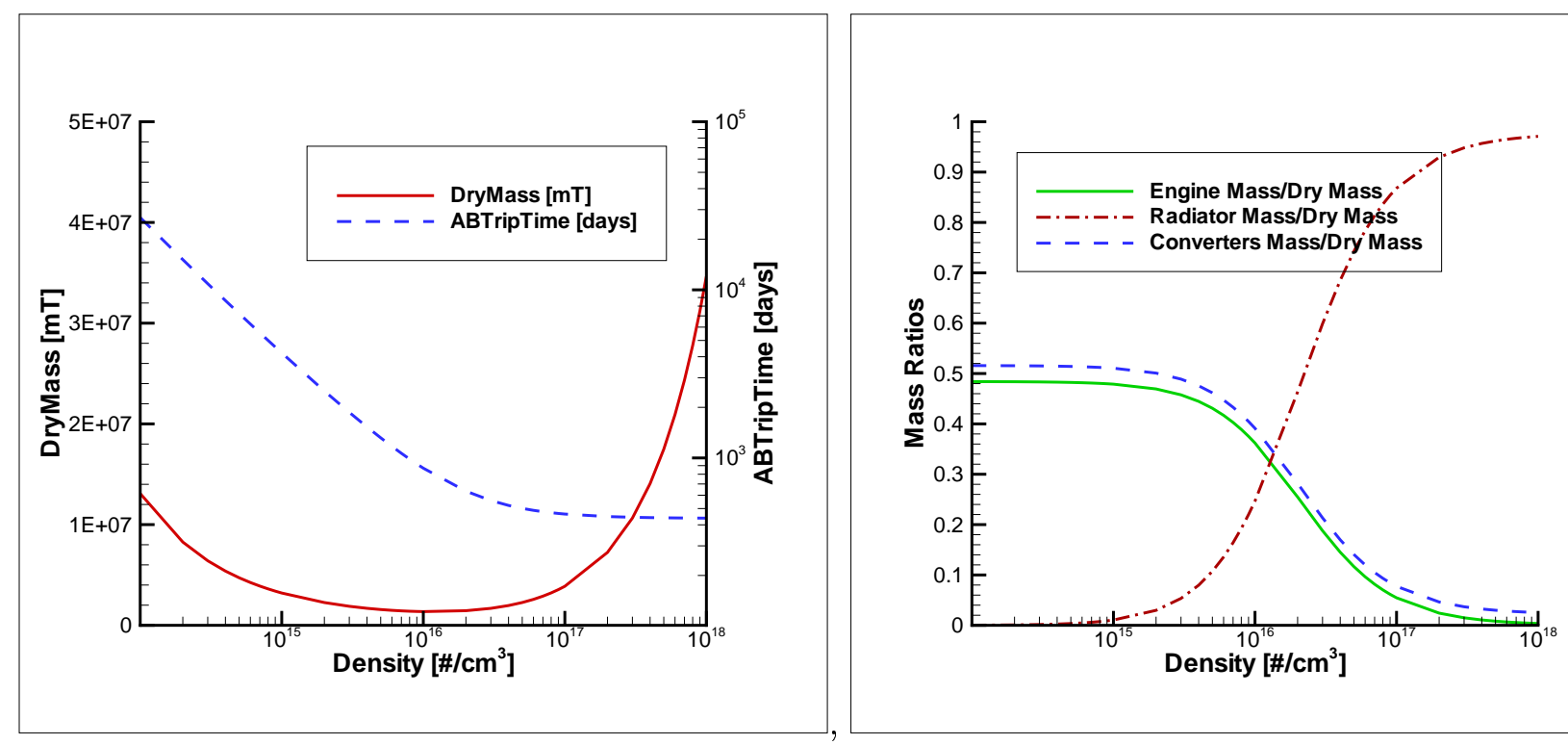

Figure 2: System mass, trip time, and component mass fractions as a function of plasma density.
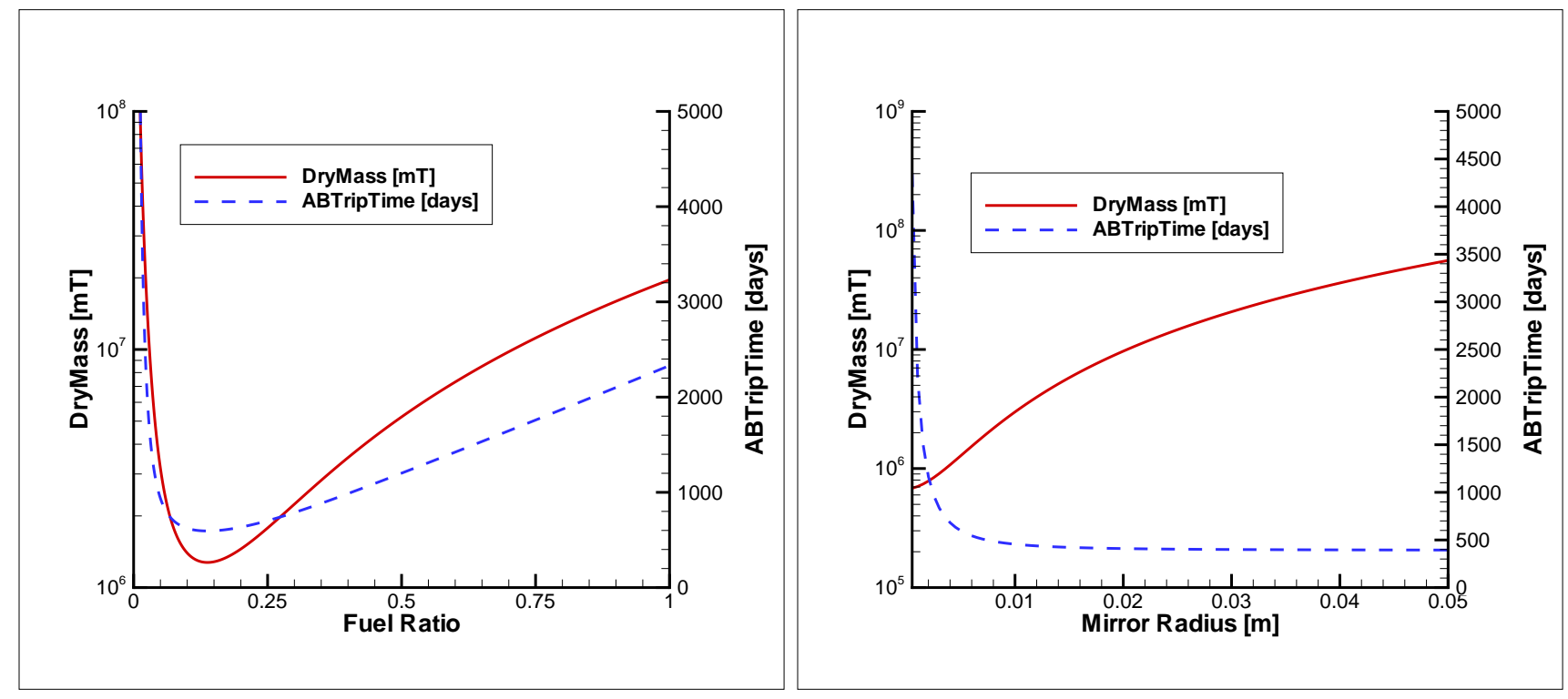

Figure 3: Fuel fraction and mirror radius impact on system mass and trip time. 


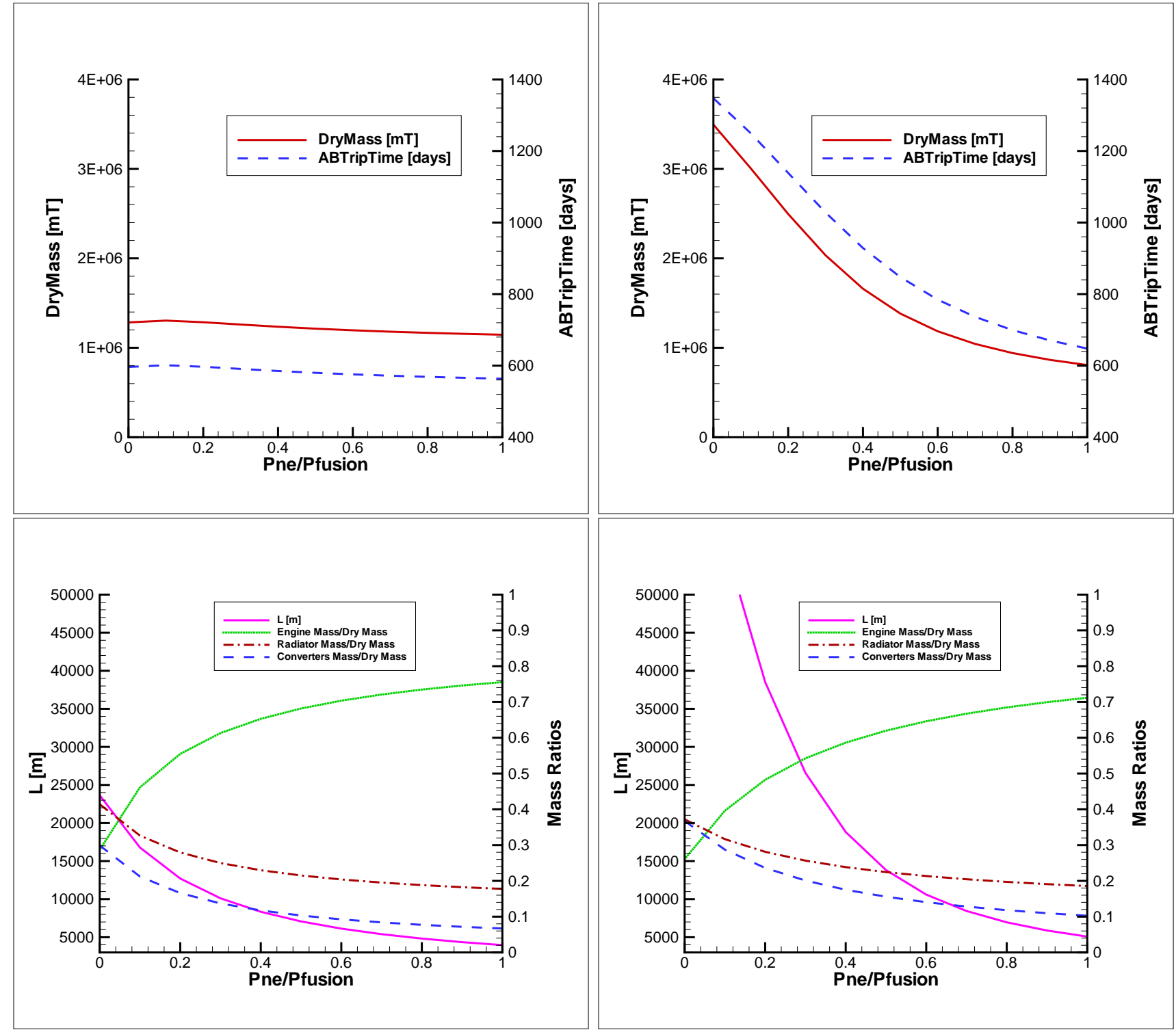

Figure 4: System parameters based on the nuclear assist fraction. $\mathrm{T}=300 \mathrm{keV}$ on the left and $\mathrm{T}=160 \mathrm{keV}$ on the right. 